\title{
Assessment of Pathological Response of Breast Carcinoma in Modified Radical Mastectomy Specimens after Neoadjuvant Chemotherapy
}

\author{
Dhanya Vasudevan, ${ }^{1}$ P. S. Jayalakshmy, ${ }^{2}$ Suresh Kumar, ${ }^{3}$ and Siji Mathew ${ }^{4}$ \\ ${ }^{1}$ Department of Pathology, Pushpagiri Institute of Medical Sciences and Research Centre, Tiruvalla, Kerala 689101, India \\ ${ }^{2}$ Department of Pathology, Government Medical College, Thrissur, Kerala 680596, India \\ ${ }^{3}$ Department of Oncology, Government Medical College, Alappuzha, Kerala 688005, India \\ ${ }^{4}$ Department of Pathology, Government Medical College, Alappuzha, Kerala 688005, India
}

Correspondence should be addressed to Dhanya Vasudevan; drdhanyabinu@gmail.com

Received 30 July 2015; Revised 29 October 2015; Accepted 1 November 2015

Academic Editor: Claudio Luparello

Copyright (C) 2015 Dhanya Vasudevan et al. This is an open access article distributed under the Creative Commons Attribution License, which permits unrestricted use, distribution, and reproduction in any medium, provided the original work is properly cited.

\begin{abstract}
Aim. Paclitaxel based neoadjuvant chemotherapy regimen (NAT) in the setting of locally advanced breast cancer (LABC) can render inoperable tumor $(\mathrm{T} 4, \mathrm{~N} 2 / \mathrm{N} 3)$ resectable. The aim of this study was to assess the status of carcinoma in the breast and lymph nodes after paclitaxel based NAT in order to find out the patient and the tumor characteristics that correspond to the pathological responses which could be used as a surrogate biomarker to assess the treatment response. Materials and Methods. Clinical and tumor characteristics of patients with breast carcinoma $(n=48)$ were assessed preoperatively. These patients were subjected to modified radical mastectomy after 3 courses of paclitaxel based NAT regimen. The pathological responses of the tumor in the breast and the lymph nodes were studied by using Chevallier's system which graded the responses into pathological complete response (pCR), pathological partial response (pPR), and pathological no response (pNR). Results. Our studies showed a pCR of $27.1 \%$ and a pPR of $70.9 \%$. Clinically small sized tumors $(2-5 \mathrm{cms})$ and Bloom Richardson's grade 1 tumors showed a pCR. Mean age at presentation was 50.58 yrs. $79.2 \%$ of cases were invasive ductal carcinoma NOS; only $2.1 \%$ were invasive lobular carcinoma, their response to NAT being the same. There was no downgrading of the tumor grades after NAT. Ductal carcinoma in situ and lymphovascular invasion were found to be resistant to chemotherapy. The histopathological changes noted in the lymph nodes were similar to that found in the tumor bed. Discussion and Conclusion. From our study we conclude that histopathological examination of the tumor bed is the gold standard for assessing the chemotherapeutic tumor response. As previous studies have shown pCR can be used as a surrogate biomarker to assess the tumor response.
\end{abstract}

\section{Introduction}

Breast carcinoma is the most common non-skin malignancy in women second to lung cancer as a cause of cancer deaths.

It has been estimated that out of all new cancer cases detected, $25 \%$ cases are breast carcinoma which also accounts for $15 \%$ of all cancer deaths among females [1]. According to GLOBOCAN 2012, the estimated cases of breast cancer worldwide are 1,676,600 with 521,900 deaths. In the developing countries the estimated new cases related to breast cancer were 882,900 and deaths were 324,300 [1]. The surveillance epidemiology and end results programme (SEER), India, Stat
Fact Sheet states that estimated new cases of breast cancer in 2015 are $231840,14 \%$ of all new cancer cases detected, and the estimated deaths were $40290,6-8 \%$ of all cancer deaths [2].

The number of new cases in India, as per GLOBOCAN 2000 , was 11,5251 , with the mortality rates being 53592 [3]. These data clearly indicate that there has been an increase in incidence of breast cancer.

The addition of adjuvant chemotherapy to standard breast carcinoma treatment has shown improving its outcome substantially [4]. The arguments offered are that the neoadjuvant chemotherapy (NAT) downstages the tumor, enables to monitor the treatment efficacy and makes it possible to 
detect and treat the micrometastasis $[5,6]$. It is possible to predict the prognosis in these patients on an individual basis by assessing the morphological parameters like a decrease in the tumor volume and inflammatory cell response.

In general, the histological responses to systemic chemotherapy can be correlated with the extent of clinical responses [7].

A study conducted by Montagna et al., 2010, states that pathological complete response (pCR) to neoadjuvant chemotherapy is the best predictor of overall survival (OS) [8].

The pathological response to paclitaxel was studied by Krishnan et al., where pCR was used as a surrogate marker for assessing the overall survival. They found that the overall pCR rate was $13.7 \%$ but with combination chemotherapy of anthracyclines and taxanes, higher rates (14.2\%) could be attained [9].

We used Chevallier's system to assess the pathological responses to paclitaxel; our study showed a pathological complete response (pCR) in $27.1 \%$ of cases and a partial pathological response (pPR) in 70.9\%.

The study conducted by Kulka et al. which aimed to study the breast cancer subtypes likely to respond to NAT also used the Chevallier system; 13 out of 92 cases showed a pCR (14.1\%). Their study also states that pCR was associated with better OS $(p=0.050)[10]$.

The greatest histopathological alterations are usually found in patients who appear to have a complete resolution of their neoplasm clinically $[11,12]$.

A study conducted by Vinnicombe et al. reported that out of 8 patients who were reported to have a complete resolution as per mammography 5 had residual neoplasm [13].

Prognosis of the patient in terms of 5-year survival rates is related to the completeness of the response and it is favourable for those patients in whom there was the least evidence of the residual neoplasm after NAT.

Thus, the histopathological examination of the tumor bed would be the gold standard to decide the presence of neoplasm after NAT.

\section{Materials and Methods}

Eligibility criteria in this study were patients $(n=48)$ with locally advanced breast cancer with axillary lymph node involvement and absence of distant metastasis.

Cases of previous lumpectomy were excluded from the study. Consent for the study was obtained from the Ethical Committee.

In this study, all patients were diagnosed to have breast carcinoma by either cytology or trucut biopsy. Prior to being subjected to chemotherapy the clinical data and the pretherapeutic clinical size of the tumor were recorded in all cases. Prechemotherapeutic tumor grading was done either from cytology smears or trucut biopsy sections (Figure 2).

The histological grading was done by Bloom Richardson grading system. The cytological grading was done by Robinson's method.
TABLE 1: Bloom Richardson grading.

\begin{tabular}{lccc}
\hline & Score 1 & Score 2 & Score 3 \\
\hline $\begin{array}{l}\text { Tubule formation } \\
\begin{array}{l}\text { Nuclear } \\
\text { pleomorphism }\end{array}\end{array}$ & Mild & Moderate & Marked \\
Mitotic figures & $<7 / 10 \mathrm{HPF}$ & $8-14 / 10 \mathrm{HPF}$ & $>15 / 10 \mathrm{HPF}$ \\
\hline
\end{tabular}

Patients received 3 courses of paclitaxel based chemotherapy (adriamycin, cyclophosphamide, and paclitaxel) and underwent modified radical mastectomy with axillary lymph node dissection (MRM).

The postchemotherapeutic changes were studied from the MRM specimens. The tumor was studied from paraffin embedded H\&E sections obtained from the tumor bed. In complete responders, the tumor bed was identified from the areas of fibrosis by multiple sampling. In partial responders and nonresponders, the tumor was evident grossly or microscopically and adequate sampling of the area was done. Tumor grade, histological type, and response to chemotherapy (by Chevallier's method) were assessed.

Bloom Richardson Grading. See Table 1:

Grade 1: score of 3-5 (well differentiated).

Grade 2: score of 6-7 (moderately differentiated).

Grade 3: score of 8-9 (poorly differentiated).

Cytological Grading from the FNAC Smears Was Done by Robinson's Grading System (Figures 1(a) and 1(b)). See Table 2:

Grade 1: score of 6-11.

Grade 2: score of 12-14.

Grade 3: score of $15-18$

2.1. Assessment of the Pathological Tumor Response. The histopathological evidence of the chemotherapeutic response was graded from the $\mathrm{H} \& \mathrm{E}$ sections on the basis of the parameters used by Chevallier in his study [14].

According to the Chevallier system we have the following:

pCR (pathological complete response): it was defined as the disappearance of all the tumor or DCIS in breast with no invasive carcinoma and negative lymph nodes.

pPR (pathological partial response): it was defined as presence of invasive carcinoma with stromal alterations.

pNR (pathological no response): it was defined as little modification in the original tumor appearance.

Only the invasive carcinomas and the lymph nodes were graded on the above-mentioned criteria.

The presence of lymphovascular emboli and presence of ductal carcinoma in situ were noted separately. 
TABLE 2: Robinson's grading system.

\begin{tabular}{lccc}
\hline & Score 1 & Score 2 & Score 3 \\
\hline Cellular cohesion & Clusters & Singly & $>5$ times the RBC \\
Cell size & $1-2$ times the RBC & $3-4$ times the RBC & Markedly pleomorphic \\
Cell uniformity & Monomorphic & Mild pleomorphism & Buds or tufts \\
Nuclear margins & Smooth & Folds & Clumps or cleaved \\
Nuclear chromatin & Vesicular & Granular & \\
\hline
\end{tabular}

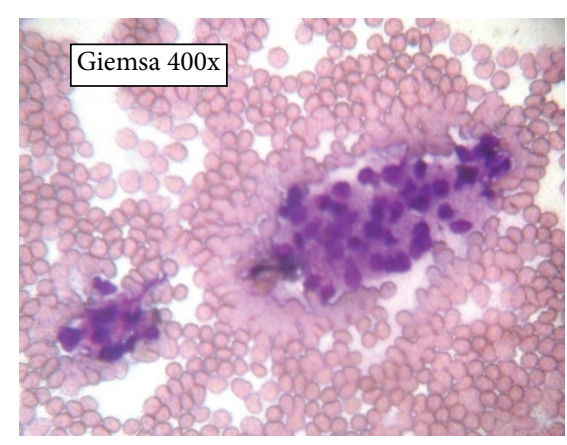

(a)

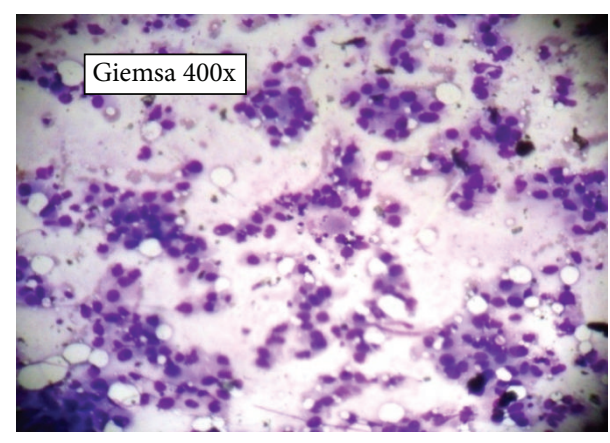

(b)

Figure 1: ( $\mathrm{a}$ and $\mathrm{b}$ ) Grade 1 tumor and Grade 2 tumor as per Robinson's grading.

\section{Analysis of the Results}

All the data were analysed with the software of SPSS v14 for windows.

Baseline sociodemographic characteristics and microscopic findings were compiled as numbers and percentages.

Response to neoadjuvant chemotherapy was also found out and expressed as numbers and percentages.

Possible risk factors affecting tumor response and lymph node response were tested using tests of proportions (Pearson's chi square test and Fischer's exact test whichever was applicable) and $p$ value was calculated. Odd's ratio and $95 \%$ confidence interval CI were also calculated from the data.

\section{Results}

In our study, majority of the patients were of the age group between 40 and 59 yrs, the mean age of presentation being 50.58 yrs (with a standard deviation of 10.94). All the 48 cases studied were females. Eight cases (16.7\%) had a positive family history for breast cancer. Majority of the cases had a clinical size of $2 \mathrm{~cm}-5 \mathrm{~cm}$ at the time of presentation $(66.7 \%)$, mean clinical size being $3.75 \mathrm{~cm}$ (with a standard deviation of 2.36) (Table 3).

Microscopic findings showed that most of the cases of pPR were of the invasive ductal carcinoma NOS (79.2\%), the rest being invasive lobular carcinoma (2.1\%) (Table 4$)$.

In the postoperative grading of the tumor, based on Bloom Richardson's grading system 13 cases of pPR were of grade $1(27.1 \%), 21$ cases were grade 2 tumors (43.8\%), and only 1 case was grade 3 tumor (2.1\%). 13 cases showed a pCR (27.1\%) (Table 4).
TABLE 3: Baseline sociodemographic characteristics.

\begin{tabular}{lcc}
\hline Characteristic & Number & Percentage \\
\hline Age & 5 & \\
$\quad$ Up to 39 & 32 & 10.4 \\
40 to 59 & 11 & 66.7 \\
$\quad 60$ and above & & 22.9 \\
Sex & 48 & 100 \\
$\quad$ Female & 0 & 0 \\
$\quad$ Male & & \\
Family history & 8 & 16.7 \\
$\quad$ Yes & 40 & 83.3 \\
$\quad$ No & & \\
Specimen size & 5 & 10.4 \\
$\quad$ Up to 2 cm & 32 & 66.7 \\
2 cm to $5 \mathrm{~cm}$ & 11 & 22.9 \\
$\quad$ More than $5 \mathrm{~cm}$ & & \\
Pre-op FNA grade (Robinsons) & 21 & 43.8 \\
$\quad$ Grade 1 & 27 & 56.2 \\
$\quad$ Grade 2 &
\end{tabular}

Ductal carcinoma in situ (DCIS) was noted in 13 cases (27.1\%) (Table 4). Lymphovascular invasion was noted in 13 cases $(27.1 \%)$ (Table 4$)$.

When the tumor response to NAT was analysed we found that 13 cases $(27.1 \%)$ showed a pathological complete response whereas 35 cases $(72.9 \%)$ showed a pathological partial response (Table 5).

When the possible risk factors for a pathological partial response were analysed it was found that 27 cases were of the age group up to 59 yrs (73\%) and 9 cases were 60 yrs of age 


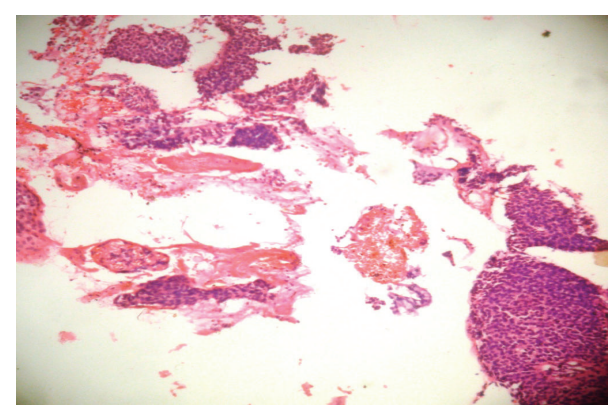

(a)

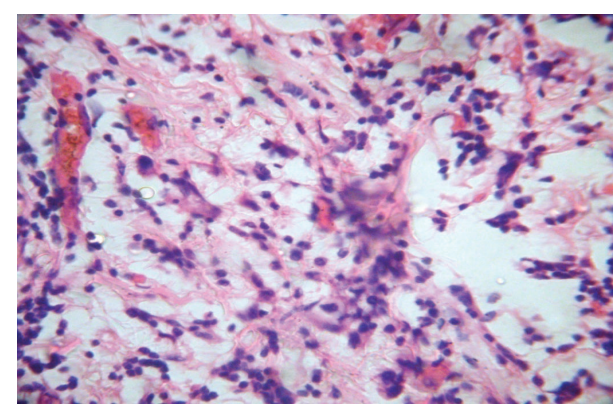

(b)

Figure 2: (a) Photomicrographs of trucut biopsy before NAT (H\&E $\times 100)$. (b) Same case after receiving NAT showing a pCR $($ H\&E $\times 400)$.

TABlE 4: Microscopic findings on examination of the resected specimen.

\begin{tabular}{lcc}
\hline Characteristic & Number & Percentage \\
\hline Type of carcinoma & & \\
pCR & 9 & 18.8 \\
Invasive ductal carcinoma NOS & 38 & 79.2 \\
Invasive lobular carcinoma & 1 & 2.1 \\
Post-op grade (Bloom Richardson) & & \\
$\quad$ Grade 0 & 13 & 27.1 \\
Grade 1 & 13 & 27.1 \\
Grade 2 & 21 & 43.8 \\
Grade 3 & 1 & 2.1 \\
Ductal carcinoma in situ (DCIS) & & \\
Yes & 13 & 27.1 \\
No & 35 & 72.9 \\
Lymph node invasion & & \\
Yes & 13 & 27.1 \\
No & 35 & 72.9 \\
\hline
\end{tabular}

TABlE 5: Percentage of tumors with the tumor responses and frequencies.

\begin{tabular}{lcc}
\hline Grades & Frequency & Percentage \\
\hline $\begin{array}{l}\text { pCR } \\
\text { pPR }\end{array}$ & 13 & $27.1 \%$ \\
Gr1 & 13 & $27.1 \%$ \\
pPR & & \\
Gr2 & 21 & $43.8 \%$ \\
pPR & & \\
Gr3 & 1 & $2.1 \%$ \\
\hline Total & 48 & $100 \%$ \\
\hline
\end{tabular}

and above (81.8\%). $62.5 \%$ had a positive family history. In 26 cases the preoperative clinical size was up to $5 \mathrm{~cm}(70.3 \%)$ and in 10 cases $(90.9 \%)$ the clinical size was more than $5 \mathrm{~cm}$. The preoperative cytological grading showed that 22 cases $(81.5 \%)$ were of grade 2 tumors whereas 14 cases $(66.7 \%)$ were of grade 1 tumors (Table 6).

11 cases $(84.6 \%)$ had ductal carcinoma in situ with a $p$ value of 0.348 and OR of 2.20 (0.41-11.75).

92.1\% were invasive ductal carcinoma NOS; this was statistically found to be significant with a $p$ value of $<0.001$ (Table 6).

13 cases $(100 \%)$ of pPR showed presence of lymphovascular invasion, which was statistically found to be significant with a $p$ value of 0.015 .

As listed in Table 6, the risk factors for pathological complete response showed the following results.

$27 \%$ of cases belonged to the age group below $59 \mathrm{yrs}$ and only 2 cases $(18.2 \%)$ were of the age group above 60 yrs.

$37.5 \%$ of cases had a positive family history for breast cancer. 11 cases $(29.7 \%)$ had a tumor size of less than $5 \mathrm{~cm}$ preoperatively and only $9.1 \%$ had a tumor size of above $5 \mathrm{~cm}$, which showed a $p$ value of 0.165 and $95 \%$ CI of 0.23 (0.02-2.07).

$33.3 \%$ of cases were grade 1 tumors and $18.5 \%$ were grade 2 tumors with $95 \%$ CI of 0.45 (0.12-1.71).

DCIS was present only in 2 cases with pCR (15.4\%); $p$ value when calculated was 0.348 with a confidence interval of $2.20(0.41-11.75)$.

Lymphovascular invasion was not seen in any case which showed pCR; this was found to be statistically significant with a $p$ value of 0.015 .

On analysing the risk factors affecting the lymph node response, it was noted that when the tumor size was less than $5 \mathrm{~cm}, 15$ cases $(40.5 \%)$ showed a pCR and $59.5 \%$ cases showed a pPR (95\% CI of $0.83(0.20-3.37)$ ) (Table 7$)$.

10 cases $(47.6 \%)$ that showed a pCR in the lymph nodes were grade 1 tumors and 9 cases (33.3\%) were grade 2 tumors. Of the cases that showed a pPR in the lymph nodes, 11 cases $(52.4 \%)$ were grade 1 tumors and 18 cases $(66.7 \%)$ were grade 2 tumors. $p$ value for which was 0.315 and $95 \%$ CI 0.55 (0.171.77) (Table 7).

Lymphovascular invasion was not seen in any case that showed a pCR and in 13 cases (100\%) of pPR. This data was found to be statistically significant with a $p$ value of 0.001 (Table 7). 
TABLE 6: Factors affecting the tumor response.

\begin{tabular}{|c|c|c|c|c|}
\hline Characteristic & Tumor partial response (pPR) & Tumor complete response (pCR) & $p$ & OR $(95 \% \mathrm{CI})$ \\
\hline \multicolumn{5}{|l|}{ Age } \\
\hline Up to 59 & $27(73 \%)$ & $10(27 \%)$ & \multirow{2}{*}{0.552} & \multirow{2}{*}{$0.60(0.11$ to 3.26$)$} \\
\hline 60 and above & $9(81.8 \%)$ & $2(18.2 \%)$ & & \\
\hline \multicolumn{5}{|l|}{ Family history } \\
\hline Yes & $5(62.5 \%)$ & $3(37.5 \%)$ & \multirow{2}{*}{0.371} & \multirow{2}{*}{0.48 (0.09 to 2.42$)$} \\
\hline No & $31(77.5 \%)$ & $9(22.5 \%)$ & & \\
\hline \multicolumn{5}{|l|}{ Specimen size } \\
\hline Up to $5 \mathrm{~cm}$ & $26(70.3 \%)$ & $11(29.7 \%)$ & \multirow{2}{*}{0.165} & \multirow{2}{*}{$0.23(0.02$ to 2.07$)$} \\
\hline More than $5 \mathrm{~cm}$ & $10(90.9 \%)$ & $1(9.1 \%)$ & & \\
\hline \multicolumn{5}{|l|}{ FNA grade } \\
\hline Grade 1 & $14(66.7 \%)$ & $7(33.3 \%)$ & \multirow{2}{*}{0.240} & \multirow{2}{*}{0.45 (0.12 to 1.71$)$} \\
\hline Grade 2 & $22(81.5 \%)$ & $5(18.5 \%)$ & & \\
\hline \multicolumn{5}{|l|}{ DCIS } \\
\hline Yes & $11(84.6 \%)$ & $2(15.4 \%)$ & \multirow{2}{*}{0.348} & \multirow{2}{*}{2.20 (0.41 to 11.75$)$} \\
\hline No & $25(71.4 \%)$ & $10(28.6 \%)$ & & \\
\hline \multicolumn{5}{|l|}{ Type of cancer } \\
\hline $\mathrm{pCR}$ & $1(11.1 \%)$ & $8(88.9 \%)$ & \multirow{3}{*}{$<0.001^{\#}$} & \multirow{3}{*}{-} \\
\hline Invasive ductal carcinoma NOS & $35(92.1 \%)$ & $3(7.9 \%)$ & & \\
\hline Invasive lobular carcinoma & 0 & $1(100 \%)$ & & \\
\hline \multicolumn{5}{|l|}{ Lymphovascular invasion } \\
\hline Yes & $13(100 \%)$ & 0 & \multirow{2}{*}{$0.015^{\#}$} & \multirow{2}{*}{-} \\
\hline No & $23(65.7 \%)$ & $12(34.3 \%)$ & & \\
\hline
\end{tabular}

TABLE 7: Factors affecting lymph node response.

\begin{tabular}{|c|c|c|c|c|}
\hline Characteristic & Lymph node partial response & Lymph node complete response & $p$ & OR $(95 \% \mathrm{CI})$ \\
\hline \multicolumn{5}{|l|}{ Age } \\
\hline Up to 59 & $22(59.5 \%)$ & $15(40.5 \%)$ & \multirow{2}{*}{0.804} & \multirow{2}{*}{$0.83(0.20$ to 3.37$)$} \\
\hline 60 and above & $7(63.6 \%)$ & $4(36.4 \%)$ & & \\
\hline \multicolumn{5}{|l|}{ Family history } \\
\hline Yes & $6(75 \%)$ & $2(25 \%)$ & \multirow{2}{*}{0.356} & \multirow{2}{*}{$2.21(0.39$ to 12.36$)$} \\
\hline No & $23(57.5 \%)$ & $17(42.5 \%)$ & & \\
\hline \multicolumn{5}{|l|}{ Specimen size } \\
\hline Up to $5 \mathrm{~cm}$ & $22(59.5 \%)$ & $15(40.5 \%)$ & \multirow{2}{*}{0.804} & \multirow{2}{*}{$0.83(0.20$ to 3.37$)$} \\
\hline More than $5 \mathrm{~cm}$ & $7(63.6 \%)$ & $4(36.4 \%)$ & & \\
\hline \multicolumn{5}{|l|}{ FNA grade } \\
\hline Grade 1 & $11(52.4 \%)$ & $10(47.6 \%)$ & \multirow{2}{*}{0.315} & \multirow{2}{*}{0.55 (0.17 to 1.77$)$} \\
\hline Grade 2 & $18(66.7 \%)$ & $9(33.3 \%)$ & & \\
\hline \multicolumn{5}{|l|}{ DCIS } \\
\hline Yes & $8(61.5 \%)$ & $5(38.5 \%)$ & \multirow{2}{*}{0.923} & \multirow{2}{*}{$1.06(0.28$ to 3.93$)$} \\
\hline No & $21(60 \%)$ & $14(40 \%)$ & & \\
\hline \multicolumn{5}{|l|}{ Type of cancer } \\
\hline $\mathrm{pCR}$ & $2(22.2 \%)$ & $7(77.8 \%)$ & \multirow{3}{*}{$0.012^{\#}$} & \\
\hline Invasive ductal carcinoma NOS & $27(71.1 \%)$ & $11(28.9 \%)$ & & - \\
\hline Invasive lobular carcinoma & 0 & $1(100 \%)$ & & \\
\hline \multicolumn{5}{|l|}{ Lymphovascular invasion } \\
\hline Yes & $13(100 \%)$ & 0 & \multirow{2}{*}{$0.001^{\#}$} & \multirow{2}{*}{-} \\
\hline No & $16(45.7 \%)$ & $19(54.3 \%)$ & & \\
\hline
\end{tabular}

\#Statistically significant. 


\section{Discussions and Conclusions}

5.1. Characteristics of Patients and Tumor. In our study the mean age of patients at diagnosis was $50.58 \mathrm{yrs}$ with a standard deviation of 10.94 . In a study conducted by Saxena et al. the mean age of presentation of breast cancer in the Indian population was 47.8 yrs [15] and as per the study conducted by Chin et al. the mean age at presentation was 52 yrs [16].

In our study the mean clinical tumor size at the diagnosis was $3.75 \mathrm{~cm}$, with a standard deviation of 2.36; it was also noted that the younger patients responded with a pCR (Table 3).

In the study of Chin et al. majority of the tumors presented in T2 stage (TNM staging), which is in accordance with our study. Their study also recommends that these patients could be candidates for NAT based on international standards [17]. A multivariate analysis study conducted by Galal et al. showed that initial tumor size $<5 \mathrm{~cm}$, absence of ductal carcinoma in situ, and absence of vascular invasion were the best predictors of tumor response to chemotherapy [18]. Their study states that clinically small sized tumors responded better to chemotherapeutic regime [18]. Study conducted by Adam et al. showed that extremes of age had tendency to develop a higher grade tumor. In our study there was only a single case of grade 3 tumor and the age group corresponded to $40 \mathrm{yrs}$; majority of the cases were low grade tumors even in the elderly (60-80 yrs).

5.2. Response of Tumor. Out of the total 48 cases studied there was a complete pathological response (pCR) in $27.1 \%$ of cases. $70.9 \%$ showed a partial pathological response (pPR). 58\% cases that showed a pCR were grade 1 tumors. In tumors that showed a pPR, $27.1 \%$ were grade 1 tumors, $27.1 \%$ were grade 2 tumors, and $2.1 \%$ were grade 3 tumors (Table 5 ).

Majority were grade 2 tumors out of which $81 \%$ showed pPR and $18.5 \%$ showed pCR. Out of the 21 cases of grade 1 tumors $66.7 \%$ showed $\mathrm{pPR}$ and $33 \%$ showed $\mathrm{pCR}$.

A pCR of $19-31 \%$ has been found in large prospective studies conducted by Smith et al. [19] and Baer et al. [20]; however the study conducted by Chin et al. found a lower rate of pCR of $10 \%$ [16]. Studies of Fayanju et al. showed a pCR of $40 \%$ [21].

Majority of the cases (99\%) were of invasive ductal carcinoma NOS; only 1 case of lobular carcinoma was encountered during the study. In a published series by Elston and Ellis the most common type of breast carcinoma was those of invasive duct carcinoma NOS which comprised about 40-75\% [22]. According to Galal et al. invasive duct carcinoma NOS was found in $88 \%$ of their cases and invasive lobular carcinoma was about $12 \%$ [18].

There were no differences in the tumor grades assessed prechemotherapeutically and postchemotherapeutically (Wilcoxon signed rank test of significance <0.001). Study by Frierson and Fechner noted that there were no differences in the histological grading of pretreatment and postchemotherapy surgical specimens [23].

In our study DCIS and lymphovascular invasion were found to be resistant to chemotherapy. Our study showed a strong association between lymphovascular invasion and

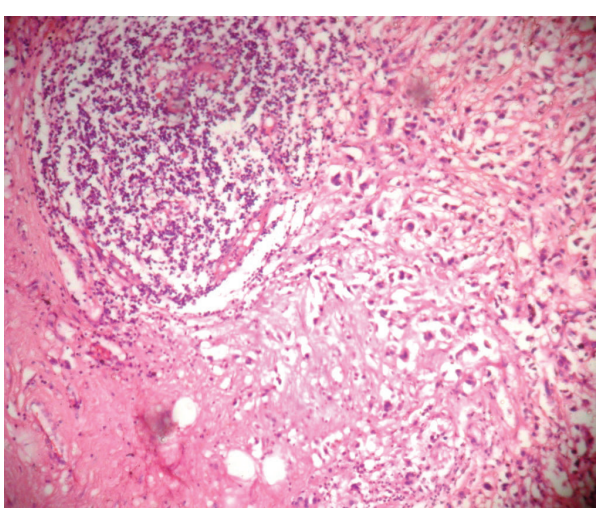

FIgURE 3: Cases of pPR showing inflammatory reaction (H\&E $\times 400)$.

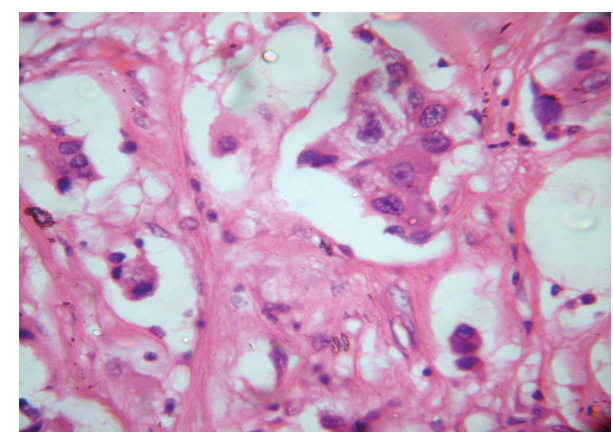

Figure 4: Photomicrograph of a case with pPR $(\mathrm{H} \& \mathrm{E} \times 400)$.

tumor response suggesting that the presence of lymphovascular invasion could indicate a poor tumor response ( $p$ value of 0.015). DCIS was consistently present in $84 \%$ of the cases with pCR indicating that it was resistant to chemotherapy. Study by Galal et al. showed that $86 \%$ of tumors with DCIS had a poor response to treatment [18].

5.3. Tumor Response in Lymph Nodes. Patients with a complete response in both breast and lymph nodes have significantly improved overall and disease-free survival [24]. In our study, in patients with pCR the histopathological findings were similar in the breast and lymph nodes which showed presence of histiocytes, giant cells, inflammatory infiltrate, and so forth.

In cases of $\mathrm{pPR}$, individual nodes in a single case showed variable responses. In these cases, nodes which showed a complete pathological response did not show any evidence of tumor but instead showed areas of fibrosis, calcification, and lymphoplasmacytic inflammatory response, whereas some nodes in the same case did not show any response to the treatment given. pCR was noted only in $7 \%$ of cases whereas pPR was noted in $93 \%$ cases. As per National Surgical Adjuvant Breast and Bowel project (NSABP 18) during a period of $9 \mathrm{yr}$ follow-up, patients with negative nodes or micrometastasis who were not treated with chemotherapy before surgery had identical survival, whereas those patients with macrometastasis had a significantly worse survival [25] (Figures 3 and 4). 


\section{Conclusion}

From our study we conclude that histopathological examination of the tumor bed is the gold standard for assessing the chemotherapeutic tumor response. Young patients with a clinically T2 tumor size and lower tumor grades were good responders to chemotherapy. Tumor grades were not downgraded after chemotherapy. $\mathrm{pCR}$ can be used as a surrogate biomarker to assess the tumor response to paclitaxel based chemotherapy regimen, as pCR has been stated as an indicator of overall survival rate by various studies. Ductal carcinoma in situ and lymphovascular emboli were resistant to chemotherapy and these patients may have a poor prognosis.

\section{Conflict of Interests}

The authors declare that there is no conflict of interests regarding the publication of this paper.

\section{Acknowledgments}

The authors would like to thank Dr. Usha Poothiode, Professor and Head of the Department, Government Medical College, Kottayam, and Dr. Krishna Balachandran, Professor of pathology, Government Medical College, Thiruvananthapuram, for their sincere help and support.

\section{References}

[1] L. A. Torre, F. Bray, R. L. Siegel, J. Ferlay, J. Lortet-Tieulent, and A. Jemal, "Global cancer statistics, 2012," CA: A Cancer Journal for Clinicians, vol. 65, no. 2, pp. 87-108, 2015.

[2] Surveillance and epidemiology and end results data, http:// seer.cancer.gov/.

[3] Globocon2008 and F. J. Shin, "Cancer incidence \& Mortality worldwide," Sections for cancer information, 2010, http://globocan.iarc.fr/.

[4] Early Breast Carcinoma Trialist Collaborative Group, "Polychemotherapy for early breast cancer: an overview of the randomised trials," The Lancet, vol. 352, no. 9132, pp. 930-942, 1998.

[5] A. Makris, T. J. Powles, S. E. Ashley et al., "A reduction in the requirements for mastectomy in a randomized trial of neoadjuvant chemoendocrine therapy in primary breast cancer," Annals of Oncology, vol. 9, no. 11, pp. 1179-1184, 1998.

[6] L. Mauriac, G. MacGrogan, A. Avril et al., "Neoadjuvant chemotherapy for operable breast cancer larger than $3 \mathrm{~cm}$ : A unicentre randomized trial with 124 month median follow up," Institute Bergonie Bordeaux group Sein(IBBGS);Ann Oncol, vol. 10, no. 1, pp. 47-52, 1999.

[7] P. P. Rosen, Rosen's Breast Pathology, chapter 43, Lippincott Williams \& Wilkins, Philadelphia, Pa, USA, 1997.

[8] E. Montagna, V. Bagnardi, N. Rotmensz et al., "Pathological complete response after preoperative systemic therapy and outcome: relevance of clinical and biologic baseline features," Breast Cancer Research and Treatment, vol. 124, no. 3, pp. 689699, 2010.

[9] Y. Krishnan, S. Al Awadi, P. S. Sreedharan, S. S. Nair, and S. Thuruthel, "Analysis of neoadjuvant therapies in breast cancer with respect to pathological complete response, disease-free survival and overall survival: 15 years follow-up data from Kuwait," Asia-Pacific Journal of Clinical Oncology, 2013.

[10] J. Kulka, A. M. Tokés, A. I. Tóth et al., "Immunohistochemical phenotype of breast carcinomas predicts the effectiveness of primary systemic therapy," Magyar Onkológia, vol. 53, no. 4, pp. 335-343, 2009.

[11] M. Briffod, F. Spyratos, M. Tubiana-Hulin et al., "Sequential cytopunctures during preoperative chemotherapy for primary breast carcinoma. Cytomorphologic changes, initial tumor ploidy, and tumor regression," Cancer, vol. 63, no. 4, pp. 631637, 1989.

[12] L. D. Feldman and G. N. Hortobagyi, "Pathological assessment of responses to induction chemotherapy in breast cancer," Cancer Research, vol. 46, pp. 2578-2581, 1986.

[13] S. J. Vinnicombe, A. D. MacVicar, R. L. Guy et al., "Primary breast cancer: mammographic changes after neoadjuvant chemotherapy, with pathologic correlation," Radiology, vol. 198, no. 2, pp. 333-340, 1996.

[14] B. Chevallier, H. Roche, J. P. Olivier et al., "Inflammatory breast cancer, pilot study of intensive induction (FEC-HD) results in a high histological chemotherapy rate," American Journal of Clinical Oncology, vol. 16, pp. 223-228, 1993.

[15] S. Saxena, B. Rekhi, A. Bansal, A. Bagga, Chintamani, and N. S. Murthy, "Clinico-morphological patterns of breast cancer including family history in a New Delhi hospital, India-a cross-sectional study," World Journal of Surgical Oncology, vol. 3, article 67, 2005.

[16] S. N. Chin, C. M. A. Green, G. M. Gordon-Strachan, and G. H. F. Wharfe, "Locally advanced breast cancer in Jamaica: prevalence, disease characteristics and response to preoperative therapy," Asian Pacific Journal of Cancer Prevention, vol. 15, no. 7, pp. 3323-3326, 2014.

[17] M. Kaufmann, G. Von Minckwitz, E. P. Mamounas et al., "Recommendations from an international consensus conference on the current status and future of neoadjuvant systemic therapy in primary breast cancer," Annals of Surgical Oncology, vol. 19, no. 5, pp. 1508-1516, 2012.

[18] A.-E. Galal, I. Iman, and B. Abdelaziz, "Response of locally advanced breast cancer to primary chemotherapy," Egyptian Journal of Surgery, vol. 26, no. 3, 2007.

[19] I. C. Smith, S. D. Heys, A. W. Hutcheon et al., "Neoadjuvant chemotherapy in breast cancer: significantly enhanced response with docetaxel," Journal of Clinical Oncology, vol. 20, no. 6, pp. 1456-1466, 2002.

[20] H. D. Bear, S. Anderson, A. Brown et al., "The effect on tumor response of adding sequential preoperative docetaxel to preoperative doxorubicin and cyclophosphamide: preliminary results from national surgical adjuvant breast and bowel project protocol B-27," Journal of Clinical Oncology, vol. 21, no. 22, pp. 4165-4174, 2003.

[21] O. M. Fayanju, I. Nwaogu, D. Jeffe, and J. Margenthaler, "Pathological complete response in breast cancer patients following neoadjuvant chemotherapy at a Comprehensive Cancer Center: the natural history of an elusive prognosticator," Molecular and Clinical Oncology, vol. 3, no. 4, pp. 775-780, 2015.

[22] C. W. Elston and I. O. Ellis, "Classification of malignant breast disease," in The Breast. Systemic Pathology, C. W. Elston and I. O. Ellis, Eds., pp. 239-247, Churchill Livingstone, Edinburgh, Scotland, 3rd edition, 1998.

[23] H. F. Frierson Jr. and R. E. Fechner, "Histologic grade of locally advanced infiltrating ductal carcinoma after treatment 
with induction chemotherapy," American Journal of Clinical Pathology, vol. 102, no. 2, pp. 154-157, 1994.

[24] L. A. Newman, H. M. Kuerer, T. L. Smith et al., "Clinical course of breast cancer patients with complete pathologic primary tumor and axillary lymph node response to doxorubicinbased neoadjuvant chemotherapy," American Journal of Clinical Oncology, vol. 17, no. 2, pp. 460-469, 1999.

[25] E. R. Fisher, J. Wang, J. Bryant, B. Fisher, E. Mamounas, and N. Wolmark, "Pathobiology of preoperative chemotherapy: findings from the National Surgical Adjuvant Breast and Bowel Project (NSABP) protocol B-18," Cancer, vol. 95, no. 4, pp. 681695, 2002. 


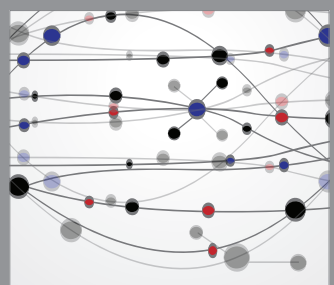

The Scientific World Journal
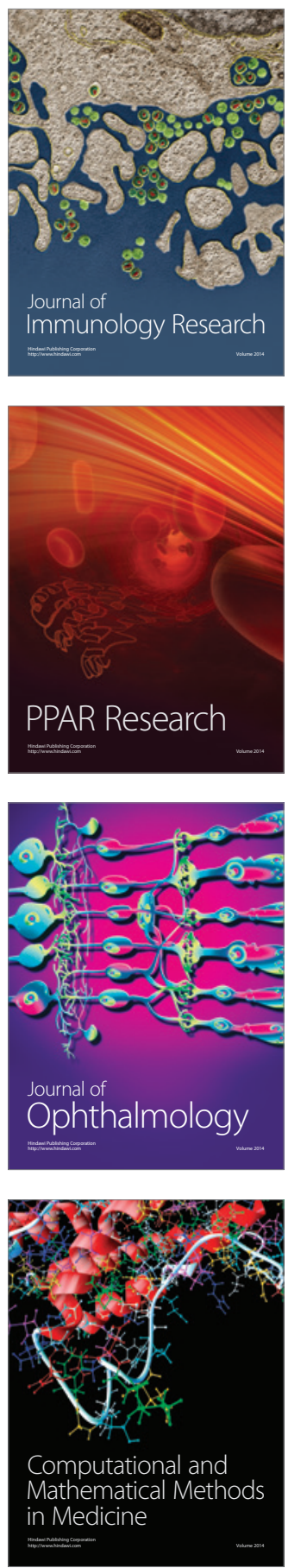

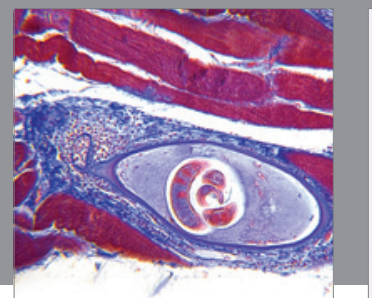

Gastroenterology

Research and Practice
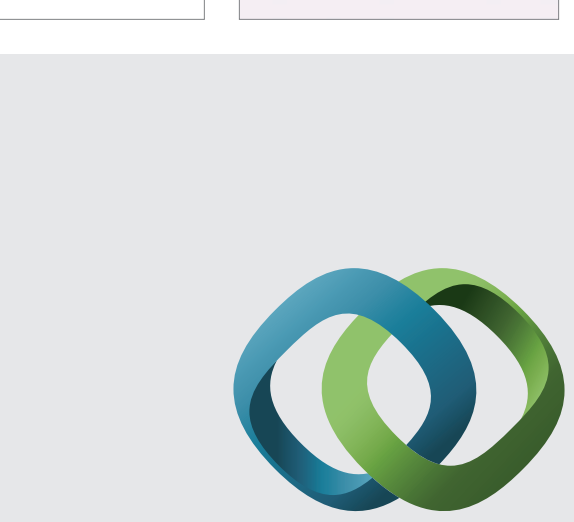

\section{Hindawi}

Submit your manuscripts at

http://www.hindawi.com
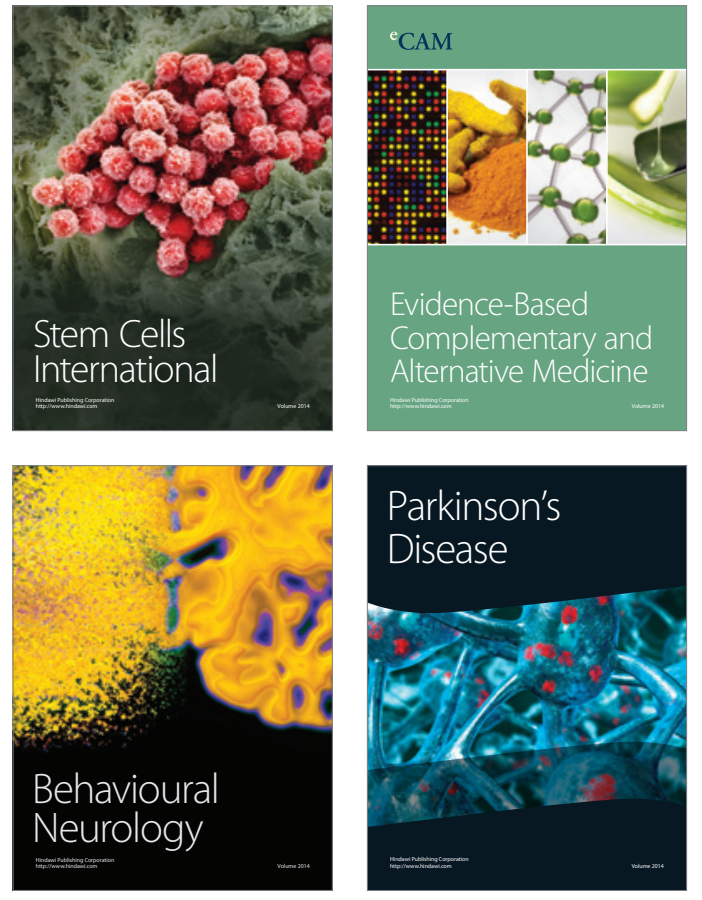
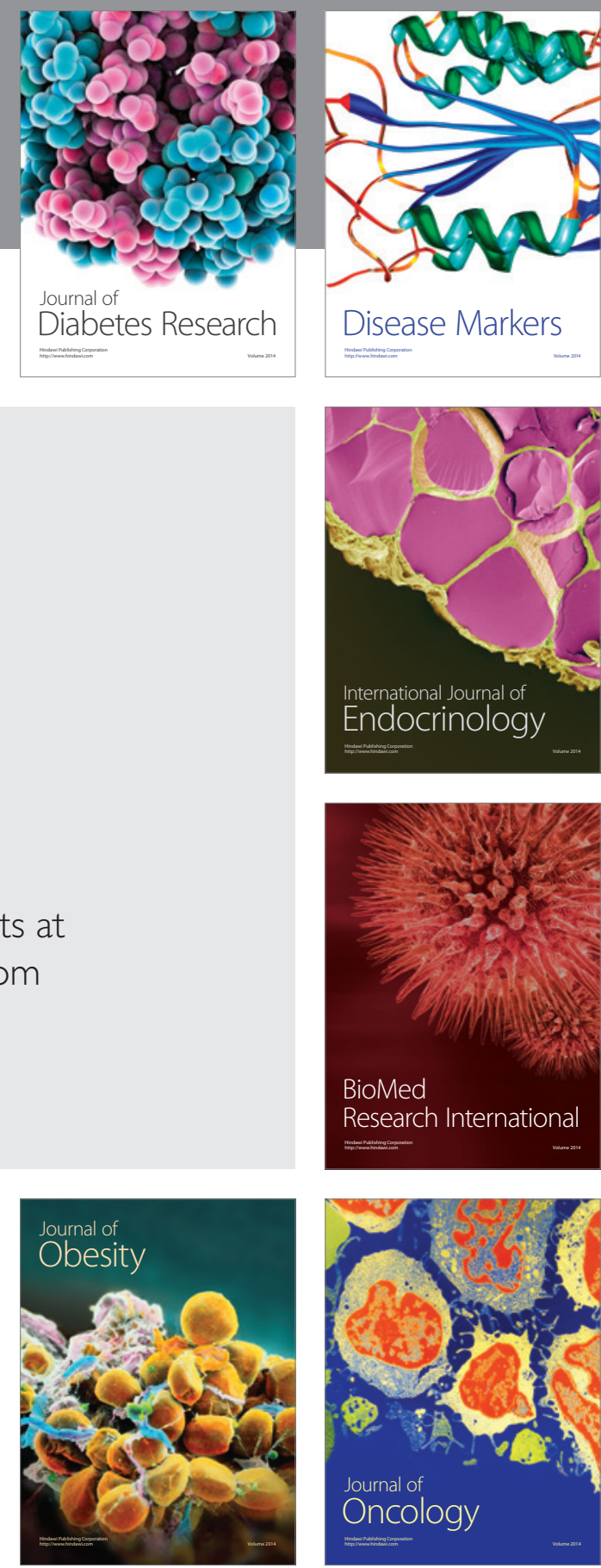

Disease Markers
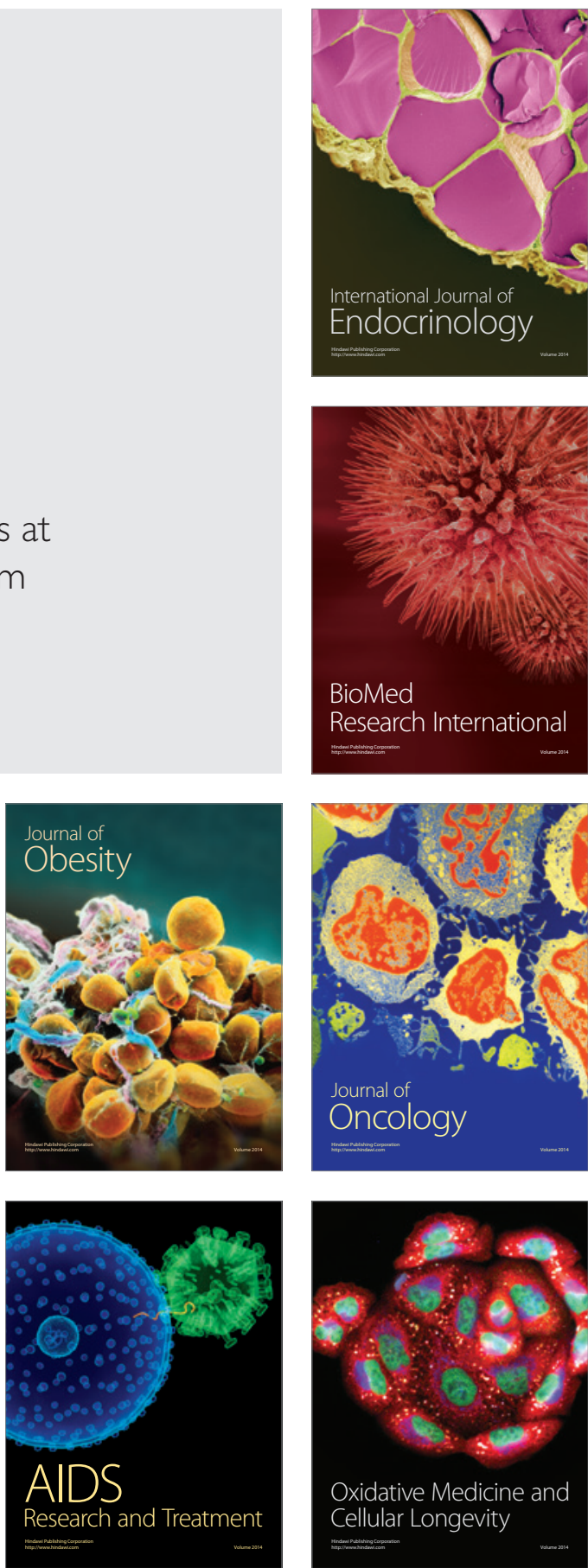